\title{
Dementia of frontal lobe type and motor neuron disease. A Golgi study of the frontal cortex
}

\author{
I Ferrer, C Roig, A Espino, G Peiro, X Matias Guiu
}

\begin{abstract}
Neuropathological findings in a 38 year old patient with dementia of frontal lobe type and motor neuron disease included pyramidal tracts, myelin pallor and neuron loss, gliosis and chromatolysis in the hypoglossal nucleus, together with frontal atrophy, neuron loss, gliosis and spongiosis in the upper cortical layers of the frontal (and temporal) lobes. Most remaining pyramidal and nonpyramidal neurons (multipolar, bitufted and bipolar cells) in the upper layers (layers II and III) of the frontal cortex (area B) had reduced dendritic arbors, proximal dendritic varicosities and amputation of dendrites as revealed in optimally stained rapid Golgi sections. Pyramidal cells in these layers also showed depletion of dendritic spines. Neurons in the inner layers were preserved. Loss of receptive surfaces in neurons of the upper cortical layers in the frontal cortex are indicative of neuronal disconnection, and are "hidden" contributory morphological substrates for the development of dementia.
\end{abstract}

Motor neuron disease associated with dementia with or without Parkinsonism is common on the island of Guam and other areas in the western Pacific region. ${ }^{1}$ Patients have large numbers of neurofibrillary tangles in the hippocampus, cerebral cortex and several nuclei of the brainstem, together with typical lesions of amyotrophic lateral sclerosis (ALS). Motor neuron involvement in patients with dementia can occur in Alzheimer's disease (AD), Pick's disease, Creutzfeldt-Jakob disease (CJD), Shy-Drager syndrome, olivoponto-cerebellar atrophy and other degenerative diseases. ${ }^{2}$ There is a third group of patients with classic ALS associated with dementia which includes sporadic and familial cases and patients with ALS and dementia and Parkinsonism. ${ }^{23}$ Clinically these patients suffer from dementia with predominant frontal signs. Histologically all of them have loss of nerve cells and status spongiosus in the upper cortical layers of the frontal and temporal lobes, whereas involvement of the substantia nigra, caudate/putamen, thalamus, other nuclei of the brainstem and sensitive spinal cord fascicles may be found in some cases. We report on the structure of neurons, revealed with the Golgi method, in the frontal cortex of a patient with dementia and motor neuron disease.

\section{Case report}

The patient was a 38 year old woman who had had slowly progressing deterioration of mental functions in the previous year. There was no family history of neurological disease. She had been working as a teacher and had given birth to eight healthy children. She first noticed frequent spelling errors and mistakes in calculating, together with slowness in thinking, repetitive acts and memory loss. The neurological examination revealed, in addition, a positive jaw jerk and snout reflex, as well as grasping. Plantar responses were flexor. Analytical tests in blood, urine and CSF, thyroid hormone and treponemal tests were normal or negative. CT scans showed slight frontal atrophy; EEC was normal. At this time, EMG examination did not reveal abnormalities.

In the following months the patient developed severe dysphagia and amyotrophy of the interoseous muscles of the hands. Fasciculations of the tongue also appeared. The second EMG study, carried out one year later, revealed signs of proximal and distal denervation in all four limbs. Motor and sensory conduction velocities were normal. Some weeks later, she complained of marked atrophy of the tongue and severe difficulty in swallowing drinks and foods. Her mental status had dramatically worsened. Both the knee and ankle reflexes were present but there was no Babinski sign. The patient died of bilateral bronchopneumonia four months after the appearance of motor signs, less than two and a half years from the beginning of the neurological disease.

NEUROPATHOLOGICAL EXAMINATION

The fresh weight of the brain was $1250 \mathrm{~g}$. It was removed less than six hours after death and immediately fixed in 5\% buffered formalin for about four weeks. Small samples of the left frontal lobe cerebral cortex (area 8) were obtained on the second day of fixation, washed several times in phosphate buffer and potassium bichromate, and stained according to the rapid Golgi method. The spinal cord was not available. Atrophy of the frontal lobes was seen on 
Figure 1 Main neuropathological findings in dementia of frontal lobe type associated with motor neuron disease. $A$ )

Demyelination of the pyramidal tracts. $B$ Massive neuron loss and severe gliosis in the hypoglossal nucleus, surviving neurons have marked chromatolytic changes (arrows). C) Neuron loss, spongiosis and gliosis in layers II and III of the frontal cortex. $A:$ Kluver Barrera $\times 3$; $B:$ Nissl stain $\times 160 ; C$ : $H \mathcal{G} E \times 160$.
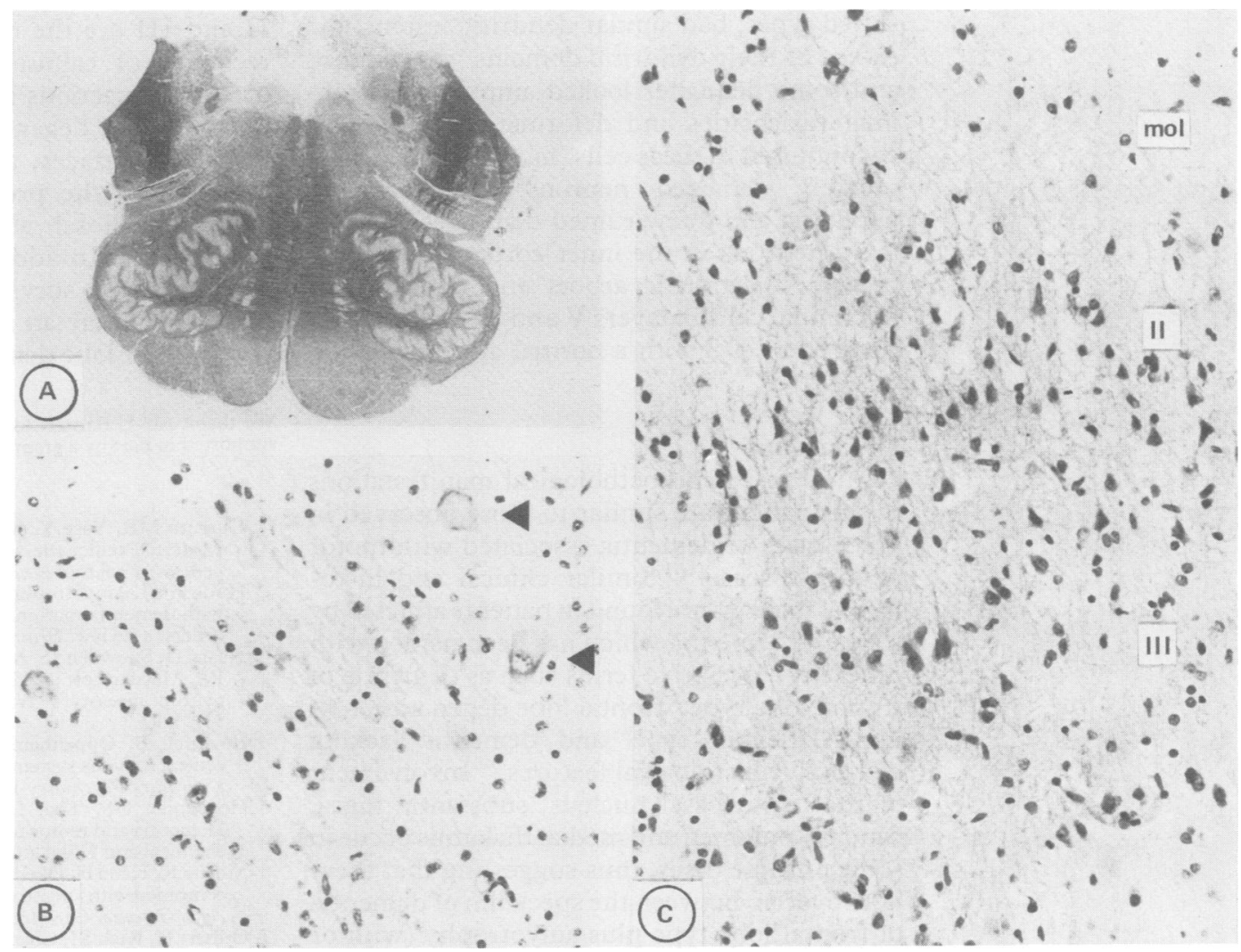
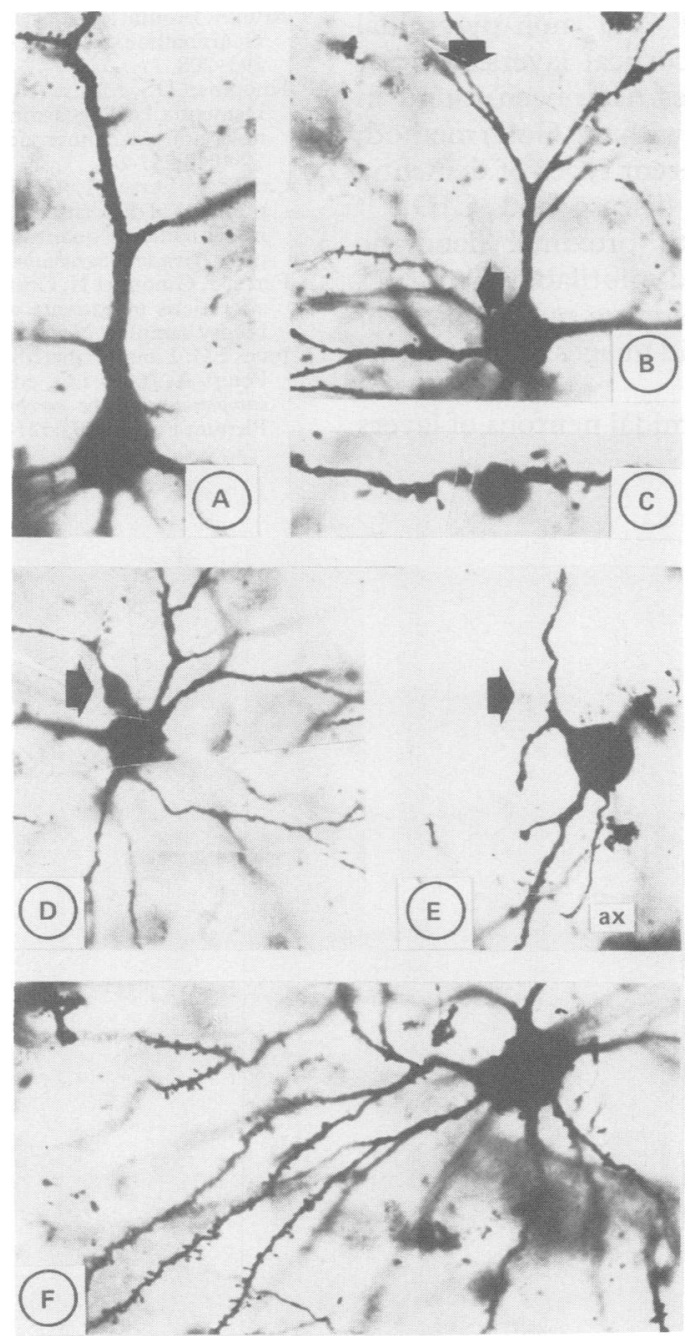

superficial examination. Stained sections revealed demyelination of the pyramidal tracts at the level of the bulbar pyramids (fig 1A). Marked neuron loss, gliosis and chromatolytic changes in the remaining neurons were present in the hypoglossal nucleus (fig 1B). The locus coeruleus and substantia nigra did not show abnormalities nor were lesions found in the striate complex, thalamus and hypothalamus. The hippocampus, subiculum and amygdaloid complex were unremarkable. Neurofibrillary tangles, senile plaques, granulovacuolar degeneration, Hirano bodies, vacuolated neurons and Pick bodies were conspicuously absent. Neuron loss, status spongiosus (coarse vacuolisation of the neuropil) and gliosis in layers II and III occurred in the cerebral cortex of the frontal lobes, and to a lesser extent of the temporal lobes as well (fig 1C). Spongiform changes (fine vacuolisation of the neuropil), as commonly seen in spongiform encephalopathies were not observed. The cerebral white matter of the frontal lobes had diffuse myelin pallor and slight gliosis.

Sections of the frontal cortex stained with the Golgi method revealed large numbers of optimally impregnated pyramidal and nonpyramidal neurons at the different cortical layers. The most striking abnormalities were localised in neurons of the upper cortical layers. Small and medium-sized pyramidal neurons exhibited reduced dendritic arbors and marked reduction in the number of dendritic spines basilar dendrites were also frequently seen in these cells (fig 2C). Non-pyramidal neurons, mainly multipolar and small bipolar and bi(DS) (fig 2A and B). Proximal varicosities of the
Figure 2 Golgi impregnated neurons in the frontal cortex. $A$ and $B$ Medium-sized and small pyramidal neurons in layer II showing severe reduction of the dendritic arbor and lack of dendritic spines (arrows). C) Proximal varicosity in a basilar dendrite of $a$ medium-sized pyramidal neuron of layer III. D Multipolar cell of layer III with reduced dendrites and displaying a proximal dendritic varicosity (arrow). E) Bipolar neuron of layer II with mutilated dendrites (arrow); ax: axon. F) Large pyramidal neuron of layer $V$ with normal basilar dendrites covered with dendritic spines. Rapid Golgi method. All but $C \times 400 ; C \times 1000$. 
tufted types, had similar dendritic lesions; the extent of their dendritic domains was reduced and some dendrites looked amputated. Proximal varicosities and deformations were also encountered in these cells (fig 2D and E). Most severely damaged neurons had shrunken perykaria and a few ruined dendrites. In contrast, neurons in the inner cortical layers had preserved dendritic arbors and dendrites of pyramidal cells in layers V and VI were largely covered by DS with a normal appearance (fig 2F).

\section{Discussion}

Clinical and neuropathological manifestations in this patient are similar to those observed in other cases of dementia associated with motor neuron disease. ${ }^{2-7}$ Similar clinical and histological findings are found in patients afflicted by a type of dementia which has been named with different descriptive terms such as dementia of frontal lobe type, ${ }^{8}$ frontal lobe degeneration of non-Alzheimer type ${ }^{9}$ and dementia lacking distinctive histological features. ${ }^{10}$ Involvement of the hypoglossal nucleus, substantia nigra, caudate/putamen and medial thalamus occur in some of these cases, thus suggesting that there is an overlap between the spectrum of dementia of frontal lobe type plus amyotrophy (with or without Parkinsonism) and ALS plus dementia (with or without Parkinsonism).

Golgi impregnations of the frontal cortex in our case show striking morphological abnormalities in pyramidal and non-pyramidal neurons of the upper cortical layers. Similar morphological anomalies have been found in biopsy samples, stained with the Golgi method, from patients with different types of dementia including $\mathrm{AD}$, Pick's disease and CJD. ${ }^{112}$ Shrunken neurons with proximal dendritic swellings, tortuous and mutilated dendrites and lacking DS are therefore not artifacts of delayed fixation but vivid images of degenerating cells.

It is known that pyramidal neurons of layers
II and III are the main source and the main recipient of callosal and ipsilateral corticocortical projections in the primate brain. ${ }^{13}$ For this reason, degeneration of the neuronal receptive surfaces, as seen in our case, is an indicator of the progressive loss of corticocortical (callosal and ipsilateral) and local connections. In addition to neuron loss, disconnection of surviving cells in the frontal cortex may play an important role in patients with frontal lobe dementia.

We thank Mr L Barnett for linguistic advice. This work was supported in part by a grant FISss $90 \mathrm{E} 1263$.

1 Garruto MR, Yase Y. Neurodegenerative disorders of the western Pacific: the search for mechanisms and pathogenesis. Trends in Neurosciences 1986;9:368-74.

2 Hudson AJ. Amyotrophic lateral sclerosis and its association with dementia, parkinsonism and other neurological disorders: a review. Brain 1981;104:217-47.

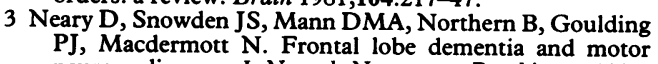
neuron disease. J Neurol Neurosurg Psychiatry 1990; 53:23-32.

4 Brownell B, Oppenheimer DR, Trevor Hughes J. The central nervous system in motor neuron disease. $J$ Neurol Neurosurg Psychiatry 1970;33:338-57.

5 Horoupian DS, Thal L, Katzman R, Terry RD, et al. Dementia and motor neuron disease: morphometric, biochemical and Golgi studies. Ann Neurol 1984;16:305-13.

6 Morita K, Kaita H, Okeda T, Namba M. Presenile dementia combined with amyotrophy: a review of 34 Japanese cases. Arch Gerontol Geriatry 1987;6:263-77.

7 Gilbert JJ, Kish SJ, Chang LJ, Hornykiewicz O. Dementia parkinsonism and motor neuron disease: Neurochemica and neuropathological correlates. Ann Neurol 1988 24:688-91.

8 Neary D, Snowden JS, Northern B, Goulding P. Dementia of frontal lobe type. J Neurol Neurosurg Psychiatry 1988;51:353-61.

9 Brun A. Frontal lobe degeneration of non-Alzheimer type. I. Neuropathology. Arch Gerontol Geriatry 1987;6: 193-208.

10 Knopman DS, Mastri AR, Frey WH, Sung JH, Rustan T. Dementia lacking distinctive histologic features: a common non-Alzheimer degenerative dementia. Neurology mon non-Alzh

11 Catalá I, Ferrer I, Galofré E, Fábregues I. Decreased numbers of dendritic spines on cortical pyramidal neurons in dementia. A quantitative Golgi study on biopsy samples. Human Neurobiology 1988;6:255-9.

12 Ferrer I, Guionnet N, Cruz-Sanchez F, Tuñón T. Neuronal alterations in patients with dementia: a Golgi study on biopsy samples. Neurosci Let 1990;114:11-16.

13 Jones EG. Laminar distribution of cortical efferent cells. In: Peters A, Jones EG, eds. Cerebral cortex vol 1, Cellular components of the cerebral cortex. New York, London:
Plenum Press, 1984:521-53. 